\title{
The Elementary Basic Principles of the Unified Theory of Relativity. $B_{3}^{*}$ VÁCLAV HLAVATÝ
}

Graduate Institute for Mathematics and Mechanics, Indiana University, Bloomington, Indiana

\section{Contents}

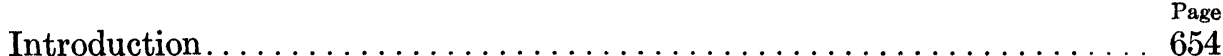

I. The tensor $N_{\lambda \mu}{ }^{\nu}$

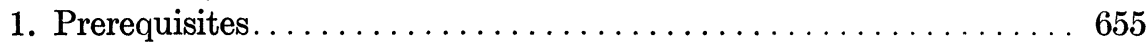

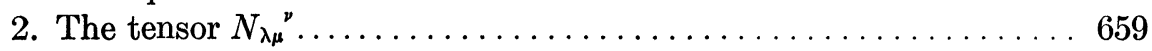

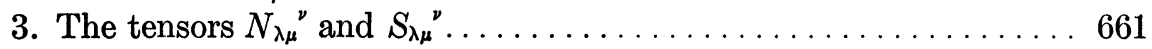

4. The identities. . . . . . . . . . . . . . . . . . . . . 662

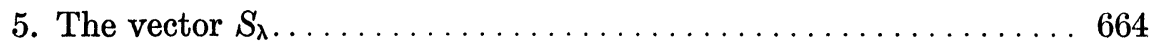

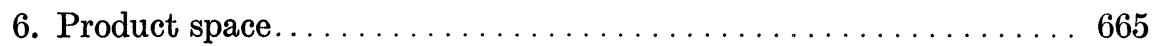

II. Degenerate cases

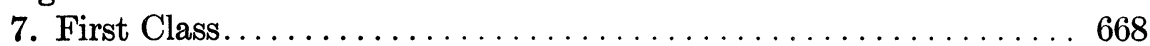

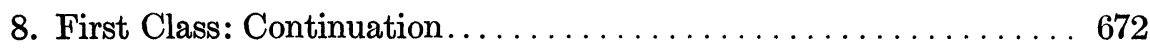

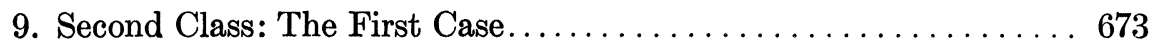

10. Second Class: The Second Case.................. 675

* Prepared under joint contract with the Office of Naval Research and the Army Office of Ordnance Research. 


\section{INTRODUCTION}

This paper is an organic continuation of the paper [4], whose results and notations will be used here without further notice. The problem to be solved is a purely algebraic one, namely, to find the solution $\Gamma_{\lambda \mu}^{\nu}$ of

$$
D_{\omega} g_{\lambda \mu}=2 S_{\omega \mu}^{\alpha} g_{\lambda \alpha}
$$

in the exceptional cases
a) $g=0$
b) $g=2$.

It will turn out that a very useful tool for this investigation is the tensor first defined for the most general case by NiJennuis [5]. In our case this tensor may be written as follows:

$$
\frac{1}{2} N_{\lambda \mu}{ }^{\nu} \stackrel{\text { def }}{=}\left(\partial_{\alpha} k_{[\lambda}{ }^{\nu}\right) k_{\mu]}{ }^{\alpha}-k_{\alpha}{ }^{\nu} \partial_{[\mu} k_{\lambda]}{ }^{\alpha} .
$$

Therefore after the necessary prerequisites we shall investigate thoroughly this tensor and in particular its relationship with $S_{\lambda \mu}{ }^{\nu}$. The corresponding results will serve in the second part as an efficient basis for the solution of the main problems of this paper. The more detailed plan of this paper may be described as follows.

After the necessary prerequisites in the first section, we deal in the second section with the geometrical properties of the tensor (0.3). In the third section we express $N_{\lambda \mu}{ }^{p}$ by means of $S_{\lambda \mu}{ }^{\nu}$. This relationship leads to some identities for $N_{\lambda \mu}{ }^{\nu}$ (Section 4). Assuming $S_{\lambda}=0$ (cf. (5.1)), ${ }^{*}$ we are then able to express $S_{\lambda \mu}{ }^{\nu}$ in terms of $N_{\lambda \mu}{ }^{\nu}$ (Section 5), and this result leads in the sixth section to the geometrical interpretation of $S_{\lambda \mu}{ }^{\nu}=0$. It turns out that this condition reduces our space-time to a Riemannian product space where every couple of eigenvectors is $X_{2}$-forming.

The second part begins with Section 7, which deals with the solution in the degenerate case ( $0.2 \mathrm{a})$ of the first class. A necessary and sufficient condition for the existence of a solution is: The eigenvectors ${ }_{3} e^{v}$ and ${ }_{4} e^{v}$ are $X_{2}$-forming (Theorem (7.3)). The condition (5.1) reduces the set of all possible solutions to a two-parametric set (Theorem (8.2)). Similar results hold also for the degenerate case (0.2a) of the second class (Section 9). The last (tenth) section deals with the solution for the degenerate case $(0.2 \mathrm{~b})$ of the second class. In particular a necessary and sufficient condition for the existence of a solution is: The eigenvectors $1 e^{\nu},{ }_{2} e^{\nu}$ are tangential vectors of geodesic lines (Theorem (10.2)). The condition (5.1) imposes here some restrictions on the field. One of these restrictions is: The eigenvectors ${ }_{1} e^{\nu}$ and ${ }_{2} e^{\nu}$ are $X_{2}$-forming (Theorem (10.6)). In all degenerate cases (with or without the restriction (5.1)) the solutions are explicitly given.

* This condition is equivalent to the first Maxwell set. $C f$. [3]. 


\section{The Tensor $N_{\lambda \mu \mu}{ }{ }^{*}$}

1. Prerequisites. (1) The equation which leads to the eigenvalues $\lambda$ of $k_{\lambda}{ }^{\nu}$ is

$$
g(\lambda) \stackrel{\text { def }}{=} \lambda^{4}+2 \lambda^{2} K+k=0 .
$$

Denoting by $D$ the scalar

$$
D=K^{2}-k,
$$

we have

$$
\begin{aligned}
& \alpha \stackrel{\text { def }}{=} \lambda_{1}=-\lambda_{2}=i \sqrt{\sqrt{D}+K} \\
& \beta \stackrel{\text { def }}{=} \lambda_{3}=-\lambda_{4}=\sqrt{\sqrt{D}-K} .
\end{aligned}
$$

The corresponding eigenvectors will be denoted by ${ }_{x} e^{\nu}$ :

$$
\lambda_{x} e^{\nu}=k_{\lambda}{ }^{\nu} e^{\lambda} .
$$

The factors of proportionality of the eigenvectors may be defined by the requirement

$$
h_{x y}=h_{\lambda \mu x} e^{\lambda} e^{\mu}
$$

where

$$
\left(\left(h_{x y}\right)\right)=\left[\begin{array}{llll}
0 & 1 & 0 & 0 \\
1 & 0 & 0 & 0 \\
0 & 0 & 0 & 1 \\
0 & 0 & 1 & 0
\end{array}\right] .
$$

* The results, introduced here without proofs, are taken from [4], [2] and [3]. We shall use the following Latin indices:

$$
\begin{array}{ll}
i, j, k, p, q, r & \text { from } 1 \text { to } 4, \\
x, y, z, t & \text { from } 1 \text { to } 4, \\
a, b, c, d & \text { from } 1 \text { to } 2, \\
e, f, g, h & \text { from } 3 \text { to } 4 .
\end{array}
$$

The indices in the last three rows $(x, \cdots, h)$ do not obey the usual summation convention. 
For the first class (i.e. $D k \neq 0$ ) all four eigenvectors are uniquely determined. In the case of the second class (i.e. $k=0, D \neq 0$ ) two eigenvalues are equal to zero and the corresponding eigenvectors belong to a pencil of vectors $e^{\nu}$ satisfying

$$
e^{\lambda} k_{\lambda}^{\nu}=0
$$

However there are exactly two of these vectors which together with the eigenvectors belonging to non-vanishing eigenvalues constitute a set of four eigenvectors satisfying (1.5).

Hence in both cases we have four linearly independent eigenvectors $i e^{\nu}$, so that there exists a unique set of four linearly independent covariant vectors ${ }^{i} e_{\lambda}$ such that
a) ${ }^{i} e_{\lambda i} e^{\nu}=\delta_{\lambda}^{\nu}$
b) ${ }^{i} e_{\lambda} e^{\lambda}=\delta_{j}^{i}$

If we denote by $\hat{e}_{\omega \mu \lambda \nu}$ the covariant indicator [3] and if $x y z t$ is an even permutation of 1234 , then we see from (1.6b) that

$$
{ }^{x} e_{\omega}=e^{-1} e_{\omega \mu \lambda \nu} e^{\mu}{ }_{z} e^{\lambda}{ }_{t} e^{\nu} \quad\left(e \stackrel{\text { def }}{=} e_{\omega \mu \lambda \nu} e^{\omega}{ }_{2} e^{\mu}{ }_{3} e^{\lambda}{ }_{4} e^{\nu}\right) .
$$

The vectors $i e^{\nu},{ }^{i} e_{\lambda}$ will serve as a frame for non-holonomic components of geometrical objects. Thus for instance the components $h_{x y}$ involved in (1.5) are non-holonomic components of $h_{\lambda \mu}$. The non-holonomic components of $k_{\lambda}{ }^{\nu}$ are

$$
k_{x}^{i}=\lambda_{x} \delta_{x}^{i}
$$

(2) Later on we shall need sums of quadratic products of eigenvectors expressed in terms of $k_{\lambda}{ }^{\nu}$ and $\delta_{\lambda}^{\nu}$. Let us denote by $\gamma$ either one of the eigenvalues $\alpha, \beta$ and put

$$
2 \sqrt{D} P_{\lambda}{ }^{\nu}(\gamma) \stackrel{\text { def }}{=} \gamma^{2} \delta_{\lambda}^{\nu}-k_{\lambda}{ }^{\alpha} k_{\alpha}^{\nu}
$$

Then the following lemma holds.

Lemma (1.1). We have

$$
\begin{aligned}
& P_{\lambda}{ }^{\nu}(\alpha)=-\sum_{f}{ }^{f} e_{\lambda} e^{\nu} \\
& P_{\lambda}{ }^{\nu}(\beta)=\sum_{a}{ }^{a} e_{\lambda_{a}} e^{\nu} .
\end{aligned}
$$

Proof. The tensor $k_{\lambda}{ }^{\nu}$ may be written

$$
k_{\lambda}{ }^{\nu}=\sum_{x} \lambda_{x}^{x} e_{\lambda x} e^{\nu}
$$

so that according to (1.6)

$$
k_{\lambda}{ }^{\alpha} k_{\alpha}{ }^{\nu}=\alpha^{2} \sum_{a}{ }^{a} e_{\lambda_{a}} e^{\nu}+\beta^{2} \sum_{f}{ }^{f} e_{\lambda f} e^{\nu} .
$$

The proof follows easily from (1.11) and (1.6a). 
Remark. $P_{\lambda \mu}=P_{\lambda}{ }^{\nu} h_{\nu \mu}$ is symmetric and moreover

$$
\begin{aligned}
-P_{\lambda \mu}(\alpha) & \left.=2{ }^{3} e_{(\lambda}{ }^{4} e_{\mu}\right) \\
P_{\lambda \mu}(\beta) & =2{ }^{1} e_{(\lambda}{ }^{2} e_{\mu)} .
\end{aligned}
$$

In the next lemma we find the corresponding skew symmetric counterpart to (1.12).

Let us denote by $\mathfrak{h}$ the determinant of $h_{\lambda \mu}$ and put

$$
\begin{gathered}
\epsilon=\operatorname{sgn} \mathfrak{e}_{\omega \mu \lambda \nu} e^{\omega}{ }_{2} e^{\mu}{ }_{3} e^{\lambda}{ }_{4} e^{\nu} \\
4 \epsilon \mathfrak{h}^{-\frac{1}{2}} \sqrt{D} Q_{\omega \mu}(\gamma) \stackrel{\text { def }}{=}-\gamma \mathfrak{e}_{\omega \mu \lambda \nu} k^{\lambda \nu}+2 \mathfrak{h}^{-\frac{1}{2}}(\gamma-\alpha-\beta) k_{\omega \mu} .
\end{gathered}
$$

Then the following lemma holds.

Lemma (1.2). We have

$$
\begin{aligned}
& Q_{\omega \mu}(\alpha)=-2{ }^{3} e_{[\omega}{ }^{4} e_{\mu]} \\
& Q_{\omega \mu}(\beta)=2{ }^{1} e_{[\omega}{ }^{2} e_{\mu]} .
\end{aligned}
$$

Proof. A simple inspection shows that

$$
\begin{aligned}
\mathfrak{e}_{\omega \mu \lambda \nu} e^{\lambda}{ }_{2} e^{\nu} & =2 \epsilon \mathfrak{h}^{-\frac{1}{2} 3} e_{[\omega}{ }^{4} e_{\mu]} \\
\mathfrak{e}_{\omega \mu \lambda \nu} e^{\lambda}{ }_{4} e^{\nu} & =2 \epsilon \mathfrak{h}^{-\frac{1}{2} 1} e_{[\omega}{ }^{2} e_{\mu]} \\
k^{\lambda \nu} & =2 \alpha_{2} e^{[\lambda}{ }_{1} e^{\nu]}+2 \beta_{4} e^{[\lambda}{ }_{3} e^{\nu]},
\end{aligned}
$$

and therefore

$$
\begin{aligned}
-\gamma e_{\omega \mu \lambda \nu} k^{\lambda \nu}+2 \epsilon \mathfrak{h}^{-\frac{1}{2}}(\gamma-\alpha & -\beta) k_{\omega \mu} \\
& =2 \epsilon \mathfrak{h}^{-\frac{1}{2}}(\alpha+\beta)\left[2(\gamma-\alpha){ }^{1} e_{[\omega}{ }^{2} e_{\mu]}+2(\gamma-\beta)^{3} e_{[\omega}{ }^{4} e_{\mu]}\right] .
\end{aligned}
$$

The tensors $P$ and $Q$ serve also for the construction of the following tensors:

$$
\begin{aligned}
& { }_{a} E_{\lambda}{ }^{\nu}={ }^{a} E_{\lambda} \stackrel{\text { def }}{=} P^{\mu \nu}(\beta)\left[h_{\mu \lambda}+(-1)^{a} Q_{\mu \lambda}(\beta)\right] \\
& { }_{f} E_{\lambda}{ }^{\nu}={ }^{f} E_{\lambda} \stackrel{\text { def }}{=}{ }^{\nu} P^{\mu \nu}(\alpha)\left[-h_{\mu \lambda}+(-1)^{f} Q_{\mu \lambda}(\alpha)\right],
\end{aligned}
$$

which occur in

Lemma (1.3). Let $\left\{\begin{array}{l}v^{\lambda} \\ w_{\nu}\end{array}\right.$ be an arbitrary vector. Then

$$
\begin{array}{lr}
v^{\lambda}{ }_{x} E_{\lambda}{ }^{\nu}=2 v^{x}{ }_{x} e^{\nu} & \left(v^{x} \stackrel{\text { def }}{=} v_{\lambda}{ }^{x} e_{\lambda}\right) \\
w_{\nu}{ }^{x} E_{\lambda}{ }^{\nu}=2 w_{x}{ }^{x} e_{\lambda} & \left(w_{x} \stackrel{\text { def }}{=} w_{\lambda} e^{\lambda}\right) .
\end{array}
$$

Proof. We have by virtue of (1.12), (1.14) and (1.6)

$$
{ }^{1} E_{\lambda}{ }^{\nu}=2{ }_{2} e^{(\mu}{ }_{1} e^{\nu)}\left[2{ }^{1} e_{(\mu}{ }^{2} e_{\lambda)}+2{ }^{3} e_{(\mu}{ }^{4} e_{\lambda)}-2{ }^{1} e_{[\mu}{ }^{2} e_{\lambda]}\right]=2{ }^{1} e_{\lambda 1} e^{\nu},
$$


and the proof of (2.16) for $x=1$ follows easily. The remaining equations (1.16) may be proved in the same way.

Remark. The equation (1.16) gives us the direction of ${ }_{x} e^{\nu}\left({ }^{x} e_{\lambda}\right)$ by means of the tensors $E$, whose construction does not require the knowledge of eigenvectors.*

(3) In order to find the solution $\Gamma_{\lambda \mu}^{\nu}$ of $(0.1)$ we shall need the scalars

$$
\lambda_{x y z}=1+\lambda_{x} \lambda_{y}+\lambda_{y} \lambda_{z}+\lambda_{z} \lambda_{x} \dagger
$$

and the tensor

$$
K_{\omega \mu \nu}=\nabla_{\omega} k_{\nu \mu}+\nabla_{\mu} k_{\omega \nu}+\nabla_{\nu} k_{\omega \mu} \ddagger .
$$

The solution $\Gamma_{\lambda \mu}^{\nu}$ of $(0.1)$ is

$$
\Gamma_{\lambda \mu}^{\nu}=\left\{\begin{array}{c}
\nu \\
\lambda \mu
\end{array}\right\}+S_{\lambda \mu}^{\nu}+2 h^{\alpha \nu} S_{\alpha(\lambda}{ }^{\beta} k_{\mu) \beta} .
$$

Here $\left\{\begin{array}{c}\nu \\ \lambda \mu\end{array}\right\}$ are Christoffel symbols of $h_{\lambda \mu}$. Hence in order to know $\Gamma_{\lambda \mu}^{\nu}$ we have to find its skew symmetric part $S_{\lambda \mu}{ }^{\nu}$. Its covariant components $S_{\lambda \mu \nu}$ satisfy the equation

$$
K_{\lambda \mu \nu}=2 X_{\lambda \mu \nu}^{\alpha \beta \gamma} S_{\alpha \beta \gamma}
$$

where $X$ is a well defined tensorial function of $k_{\lambda}{ }^{\nu \S}$. The equation (1.20) is equivalent to the following set for the non-homonomic components:

$$
\begin{array}{ll}
\text { (1.21a) } & \frac{1}{2} K_{x y z}=S_{x y z}\left(\lambda_{x y z}-\lambda_{x} \lambda_{y}\right)-S_{y z x} \lambda_{x} \lambda_{y}-S_{z x y} \lambda_{x} \lambda_{y} \\
\text { (1.21b) } & \frac{1}{2} K_{y z x}=-S_{x y z} \lambda_{y} \lambda_{z}+S_{y z x}\left(\lambda_{x y z}-\lambda_{y} \lambda_{z}\right)-S_{z x y} \lambda_{y} \lambda_{z} \\
\text { (1.21c) } & \frac{1}{2} K_{z x y}=-S_{x y z} \lambda_{z} \lambda_{x}-S_{y z x} \lambda_{z} \lambda_{x}+S_{z x y}\left(\lambda_{x y z}-\lambda_{z} \lambda_{x}\right) .
\end{array}
$$

The set of 24 equations (1.20) splits into a set of 12 equations (1.21a) for $x=z$ and four sets of three equations $(1.21 \mathrm{a}, \mathrm{b}, \mathrm{c})$ for $x, y, z \neq$. Introducing the scalar $g \stackrel{\text { def }}{=} g(1)$, i.e.

$$
g=1+2 K+k
$$

the determinant of $(1.21 \mathrm{a}, \mathrm{b}, \mathrm{c})$ (for $x, y, z \neq)$ ), namely $\lambda_{x y z}^{2}$, is different from zero if and only if (for the first class)

$$
g \neq 0 \text {. }
$$

* All that we need is $g_{\lambda \mu}$ and the eigenvalues.

$\dagger$ We are interested only in the $\lambda_{x y z}$ with at least two different indices.

$\ddagger \nabla_{\mu}$ is the symbol of the covariant derivative with respect to the Christoffel symbols of $h_{\lambda \mu}\left(=g_{(\lambda \mu)}\right)$.

$\S C f$. also [1]. 
In dealing with the second class we have to add another condition resulting from (1.21a) for $x=z$ :

$$
g-2 \neq 0 .
$$

Whenever the conditions (1.23) are satisfied then the solution of (1.21) may be written

$$
S_{x y z}=\frac{1}{2 \lambda_{x y z}}\left(K_{x y z}+3 K_{[x y z]} \lambda_{x} \lambda_{y}\right)
$$

This paper, however, is devoted to the exceptional cases characterized by (0.2), which contradicts (1.23).

The equation (1.19a) may be written in the non-holonomic frame as

$$
\Gamma_{j k}^{i}=\left\{\begin{array}{c}
i \\
j k
\end{array}\right\}+S_{j k}^{i}+2 h^{i p} S_{p(j}^{q} k_{k) q},
$$

where

$$
\left\{\begin{array}{c}
i \\
j k\}
\end{array}\right\} \stackrel{\text { def }}{=}-{ }_{j} e^{\lambda}{ }_{k} e^{u} \nabla_{\mu}{ }^{i} e_{\lambda}
$$

are non-holonomic components of Christoffel symbols.

(4) The conditions $(1.23 \mathrm{a}, \mathrm{b})$ are satisfied in the case of the third class (i.e. $D=k=0$ ) where $g=1$. Therefore the third class is excluded from our considerations*. Unless stated explicitly otherwise, the results of the first part hold for the remaining classes and even in the cases characterized by $(0.2)$.

\section{The tensor $N_{\lambda \mu}{ }^{\nu}$.}

Theorem (2.1). The non-holonomic components

$$
N_{x y}{ }^{z}={ }_{x} e^{\lambda}{ }_{y} e^{\mu} N_{\lambda \mu}{ }^{\nu}{ }^{z} e_{\nu}
$$

of $N_{\lambda \mu}{ }^{\nu}$ are

$$
\begin{gathered}
\frac{1}{2} N_{x y}{ }^{2}=\left\{\begin{array}{c}
z \\
{[x y]}
\end{array}\right\}\left(\lambda_{z}-\lambda_{x}\right)\left(\lambda_{z}-\lambda_{y}\right) \quad(x, y, z \neq) \\
N_{x y}{ }^{y}=\left(\lambda_{y}-\lambda_{x}\right) \partial_{x} \lambda_{y} .
\end{gathered}
$$

Proof. The symbol $\partial$ in (0.3) may be replaced by $\nabla$ and the equation so obtained rewritten in non-holonomic components. Substituting into the latter equation from (1.7), we obtain (2.2). This result is independent of any special value of the scalar $g$.

* The solution of (1.20) for the third class has been dealt with in [4]. $C f$. also [2]. 
Theorem (2.2). Assume

$$
\left(\lambda_{z}-\lambda_{x}\right)\left(\lambda_{z}-\lambda_{y}\right) \neq 0 .^{*}
$$

Then a necessary and sufficient condition for the eigenvectors ${ }_{x} e^{\nu},{ }_{y} e^{\nu}$ to be $X_{2}$-form. ing is

$$
N_{x y}^{2}=0
$$$$
(x, y, z \neq) \text {. }
$$

Proof. A necessary and sufficient condition for ${ }_{x} e^{\nu}$ and ${ }_{y} e^{\nu}$ to be $X_{2}$-forming is

$$
{ }_{x} e^{\lambda}{ }_{y} e^{\mu} \nabla_{[\mu}{ }^{z} e_{\lambda]}=0 \quad(x, y, z \neq 0),
$$

and this equation is equivalent to

$$
\left\{\begin{array}{c}
z \\
{[x y]}
\end{array}\right\}=0 \quad(x, y, z \neq 0)
$$

(cf. (1.24)). Equation (2.5) is by virtue of (2.3a) and (2.2a) equivalent to (2.3b).

Remark. This theorem has been proved in [5] by another method.

Theorem (2.3). A necessary and sufficient condition for the eigenvalues $\lambda_{1}, \ldots, \lambda_{4}$ to be constant is

$$
N_{x y}^{y}=0, \quad x \neq y .
$$

Proof. Let us first introduce the indices $x^{\prime}, y^{\prime}, z^{\prime}$ by the following agreement:

$$
\text { If } x=1,2,3,4, \quad \text { then } \quad x^{\prime}=2,1,4,3
$$

and similarly for $y^{\prime}, z^{\prime}$. Then we have

$$
\partial_{x} \lambda_{x^{\prime}}=-\partial_{x} \lambda_{x}
$$

so that

$$
\partial_{x} \lambda_{y}=0
$$

for all $x$ and all $y \neq x$ yields constant eigenvalues. Then the theorem follows at once for the first class. If however we are dealing with the second class, then for $\lambda_{x}=\lambda_{y}=0$ we also obtain (2.6) by virtue of (2.2b).

The equations (2.3b) and (2.6) may be rewritten in a way which does not require eigenvectors.

Theorem (2.4). Let $v^{\nu}\left(w_{\lambda}\right)$ be an arbitrary vector $\dagger$. Equations (2.3b) and (2.6) are equivalent to

$$
{ }_{x} E_{\alpha}^{\lambda}{ }_{y} E_{\beta}{ }^{\mu \nu} E_{\nu}{ }^{\gamma} N_{\lambda \mu}{ }^{\nu} v^{\alpha} v^{\beta} w_{\gamma}=0
$$

* We have $\lambda_{z}=\lambda_{x}\left(\lambda_{z}=\lambda_{y}\right)$ if and only if $\lambda_{z}=\lambda_{x}=0\left(\lambda_{z}=\lambda_{y}=0\right)$. Hence (2.3a) is automatically satisfied for the first class.

$\dagger$ By an "arbitrary vector" we mean any vector whose transvection with ${ }_{x} E_{\lambda}$ $(x=1, \cdots, 4)$ is not equal to zero. 
and

$$
{ }_{x} E_{\alpha}{ }^{\lambda}{ }_{y} E_{\beta}{ }^{\mu y} E_{\nu}{ }^{\gamma} N_{\lambda \mu}{ }^{\nu} v^{\alpha} v^{\beta} w_{\gamma}=0 \text {. }
$$

The proof follows at once from Lemma (1.3).

Remark. If in (2.3b) $x, y=3,4$ or $x, y=1,2$, this equation may be rewritten in a way which does not involve the eigenvectors and the arbitrary vectors, namely

$$
Q^{\lambda \mu}(\gamma) N_{\lambda \mu}{ }^{\alpha} P_{\alpha}{ }^{\nu}(\gamma-\alpha-\beta)=0 .
$$

The proof follows easily from (1.14) and (1.12).

\section{The tensors $N_{\lambda \mu}{ }^{\nu}$ and $S_{\lambda \mu}{ }^{\nu}$.}

Theorem (3.1). Let $D_{\mu}$ be the symbol of the covariant derivative with respect to an arbitrary connection $\Gamma_{\lambda \mu}^{\nu}$, whose skew symmetric part is $S_{\lambda \mu}{ }^{\nu}$. Put

$$
T_{\lambda \mu} \stackrel{\text { def }}{=} D_{\lambda} k_{\mu}{ }^{\nu}
$$

Then

$$
\begin{array}{r}
\frac{1}{2} N_{\lambda \mu}{ }^{\nu}=T_{\alpha[\lambda}{ }^{\nu}{k_{\mu]}}^{\alpha}-T_{[\mu \lambda]}^{\alpha} k_{\alpha}{ }^{\nu}-k_{\beta}{ }^{\nu}\left(2 S_{\alpha[\lambda}{ }^{\beta}{k_{\mu]}}^{\alpha}+S_{\lambda \mu}{ }^{\alpha} k_{\alpha}{ }^{\beta}\right) \\
-S_{\beta \alpha} k_{[\lambda}{ }^{\beta} k_{\mu]}{ }^{\alpha} .
\end{array}
$$

Proof. We have

$$
T_{\xi \eta}^{\zeta}=D_{\xi} k_{\eta}^{\zeta}=\partial_{\xi} k_{\eta}^{\zeta}-S_{\eta \xi}^{\alpha} k_{\alpha}^{\zeta}+S_{\alpha \xi}{ }^{\zeta} k_{\eta}^{\alpha}+(*)
$$

where $(*)$ denotes terms containing the symmetric part of the connection. Substituting $\partial_{\xi} k_{\eta}^{\zeta}$ from (3.3) into (0.3), we obtain (3.2).

The next auxiliary theorem, needed later on, is

Theorem (3.2). If the connection $\Gamma_{\lambda \mu}^{\nu}$ is defined by (0.1) then we have

$$
T_{\xi \eta}{ }^{\zeta}=-S_{\xi \alpha}^{\zeta}\left(\delta_{\eta}^{\alpha}-k_{\beta}{ }^{\alpha} k_{\eta}{ }^{\beta}\right)+\left(S_{\beta \xi}{ }^{\alpha} k_{\eta}{ }^{\beta}-S_{\xi \eta}{ }^{\alpha}\right)\left(\delta_{\alpha}^{\zeta}-k_{\alpha}{ }^{\zeta}\right) .
$$

Proof. Equation (0.1) yields in turn

$$
\begin{aligned}
D_{\omega} h_{\lambda \mu} & =2 S_{\omega(\mu}{ }^{\alpha} g_{\lambda) \alpha} \\
-h_{\lambda \mu} D_{\omega} h^{\lambda \nu} & =2 S_{\omega(\mu}{ }^{\alpha} g_{\lambda) \alpha} h^{\lambda \nu} \\
D_{\omega} h^{\lambda \nu} & =-2 S_{\omega(\alpha}{ }^{\gamma} g_{\beta) \gamma} h^{\beta \nu} h^{\alpha \lambda} \\
D_{\omega} k_{\lambda \mu} & =2 S_{\omega[\mu}{ }^{\alpha} g_{\lambda] \alpha} \\
D_{\xi} k_{\eta}^{\zeta} & =\left(D_{\xi} k_{\eta \lambda}\right) h^{\lambda \zeta}+k_{\eta \lambda} D_{\xi} h^{\lambda \zeta} .
\end{aligned}
$$

Substituting into (3.5e) from (3.5d) and (3.5c) one obtains (3.4). 
Theorem (3.3). If the connection $\Gamma_{\lambda \mu}^{\nu}$ is defined by (0.1), then the tensors $S_{\lambda \mu}{ }^{\nu}$ and $N_{\lambda \mu}{ }^{\nu}$ are related by

$$
N_{\lambda \mu \nu}=2 Z_{\lambda \mu \nu}^{\xi \eta \xi} S_{\xi \eta \xi}
$$

where

$$
\begin{aligned}
2 Z_{\lambda \mu \nu}^{\xi \eta \xi}=-\delta_{\nu}^{[\xi} & k_{[\mu}^{\eta \eta]} \delta_{\lambda]}^{\zeta}+k_{[\lambda}^{\beta} k_{\mu]}{ }^{[\eta} \delta_{\nu}^{\xi]} k_{\beta}{ }^{\zeta}-\delta_{\nu[\lambda}^{\zeta[\eta} k_{\mu]}{ }^{\xi]}-k_{\nu}{ }^{\zeta} k_{[\lambda}{ }^{[\eta} k_{\mu]}^{\xi]} \\
& -k_{\nu}{ }^{[\xi} \delta_{[\mu \lambda]}^{\eta] \xi}+k_{[\lambda}{ }^{\beta} \delta_{\mu]}^{[\eta} k_{\nu}{ }^{\xi]} k_{\beta}{ }^{\zeta}-k_{\nu}{ }^{\zeta} \delta_{[\mu \lambda]}^{[\xi \eta]}-\delta_{[\mu}^{[\xi} k_{\lambda]}{ }^{\eta]} k_{\beta}{ }^{\zeta} k_{\nu}{ }^{\beta} .
\end{aligned}
$$

Proof. One obtains (3.6) upon substituting into (3.2) from (3.4).

Remark. There is a basic difference between (3.2), which holds in every case and (3.6), which holds only in some cases:

Theorem (3.4). A sufficient condition for the relationship (3.6) is that equation (0.1) admit at least one solution*.

Proof. The equations (3.6) result from (0.1), (3.4) and (0.3), (3.2). The latter equations hold in every case. The equation (3.4) exists only if (0.1) is meaningful, i.e. if it admits at least one solution.

Remark. This theorem will play a paramount role in the degenerate cases, characterized by $(0.2)$.

Theorem (3.5). The relation (3.6) is equivalent to

$$
\begin{aligned}
N_{x y z}=S_{x y z}\left[\lambda_{x}+\lambda_{y}+\lambda_{z} \lambda_{x y z}+\lambda_{z}\left(1+\lambda_{x} \lambda_{y}\right]\right. & +S_{y z x}\left(\lambda_{y}+\lambda_{z}\right)\left(1-\lambda_{x}^{2}\right) \\
& +S_{z x y}\left(\lambda_{x}+\lambda_{z}\right)\left(1-\lambda_{y}^{2}\right)
\end{aligned}
$$

for arbitrary $x, y, z$.

Proof. Equation (3.6b) shows that according to (1.7) the non-holonomic components $Z_{p q r}^{i j k}$ for which $i j k$ is not a permutation of $p q r$ are equal to zero. Moreover they are skew symmetric in $i j$ as well as in $p q$. Therefore the non-holonomic form of (3.6a) is

$$
\begin{aligned}
N_{x y z} & =2 Z_{x y z}^{i j k} S_{i j k} \\
& =4 Z_{x y z}^{x y z} S_{x y z}+4 Z_{x y z}^{y z x} S_{y z x}+4 Z_{x y z}^{z x y} S_{z x y}
\end{aligned}
$$

Substituting into the non-holonomic components of $Z$ from (1.7), one obtains from (3.8) the equation (3.7).

4. The identities. Let us prove first an auxiliary

Theorem (4.1). The equations (3.6) cannot be inverted.

Proof. It is sufficient to prove that (3.7), equivalent to (3.6), cannot be inverted: For $z \neq x, x^{\prime}$ equation (3.7) yields

$$
N_{x x^{\prime} z}=\left[2 S_{x x^{\prime} z} \lambda_{z}+S_{x^{\prime} z x}\left(\lambda_{z}-\lambda_{x}\right)+S_{z x x^{\prime}}\left(\lambda_{z}+\lambda_{x}\right)\right] \lambda_{x x^{\prime} z}
$$

* A necessary and sufficient condition for (0.1) to admit exactly one solution is $g \neq 0$ for the first class and $g(g-2) \neq 0$ for the second class (cf. [2]). 


$$
\begin{aligned}
& N_{x^{\prime} z x}=\left[S_{x^{\prime} z x}\left(\lambda_{z}+\lambda_{x}\right)+S_{z x x^{\prime}}\left(\lambda_{z}+\lambda_{x}\right)\right] \lambda_{x x^{\prime} z} \\
& N_{z x x^{\prime}}=\left[S_{x^{\prime} z x}\left(\lambda_{z}-\lambda_{x}\right)+S_{z x x^{\prime}}\left(\lambda_{z}-\lambda_{x}\right)\right] \lambda_{x x^{\prime} z} .
\end{aligned}
$$

On the other hand we obtain from (3.7)

$$
N_{x y x}=2 \lambda_{x} \lambda_{x x y} S_{x y x} \quad\left(=-N_{y x x}\right) .
$$

The equations (4.2) represent all equations for non-holonomic components of $N$ with two equal indices, while (4.1) represent all equations for non-holonomic components of $N$ with three different indices. The determinant of the system (4.1)-looked upon as a system for the unknowns $S$-is equal to zero. Hence (4.1) cannot be inverted.

Theorem (4.2). The components $N_{\lambda \mu}{ }^{\nu}$ are not independent. They satisfy the condition

$$
P^{\alpha \beta}(\gamma)\left[k_{\lambda}{ }^{\mu} N_{\mu \alpha \beta}+N_{\lambda \mu(\alpha} k_{\beta}{ }^{\mu}\right]=0
$$

and consequently also

$$
k_{\lambda}{ }^{\mu} N_{\mu \alpha}{ }^{\alpha}+N_{\lambda \mu}{ }^{\alpha} k_{\alpha}{ }^{\mu}=0 .
$$

Proof. Assuming first that $g \neq 0$, we have $\lambda_{x x^{\prime} z} \neq 0$. Then the last two equations (4.1) yield

$$
\begin{aligned}
0 & =\left(\lambda_{z}-\lambda_{x}\right) N_{x^{\prime} z x}-\left(\lambda_{z}+\lambda_{x}\right) N_{z x x^{\prime}} \\
& =-\lambda_{z}\left(N_{z x^{\prime} x}+N_{z x x^{\prime}}\right)-\lambda_{x^{\prime}} N_{z x^{\prime} x}-\lambda_{x} N_{z x x^{\prime}} .
\end{aligned}
$$

This relation derived for $z \neq x, x^{\prime}$ holds also for $z=x$ or $z=x^{\prime}$ as a simple inspection shows. Hence using (1.7) we may rewrite (4.5) in the form

$$
k_{i}^{j} N_{j\left(x x^{\prime}\right)}+N_{i j(x} k_{\left.x^{\prime}\right)}^{j}=0 \text {, }
$$

where the indices $i, x$ are quite arbitrary. Hence we have by virtue of (1.12)

$$
P^{p r}(\gamma)\left(k_{i}^{j} N_{j p r}+N_{i j(p} k_{r)}{ }^{j}\right)=0,
$$

and this equation is equivalent to (4.3). Equation (4.4) is then an immediate consequence of (4.3) and (1.12). The remaining part of the proof (for $g=0$ ) will be given with the proof of

Theorem (4.3). The condilions (4.3) and (4.4) do not impose any new restriction on $k_{\lambda}{ }^{\nu}$, for these conditions are identically satisfied.

Proof. It is obviously sufficient to prove that (4.5) is an identity. Put

$$
A(x, z) \stackrel{\text { def }}{=}\left(\lambda_{z}-\lambda_{x}\right) N_{x^{\prime} z x}-\left(\lambda_{z}+\lambda_{x}\right) N_{z x x^{\prime}} .
$$

Then we have by virtue of (1.5)

$$
A(x, z)=\left(\lambda_{z}-\lambda_{x}\right) N_{x^{\prime} z}^{x^{\prime}}-\left(\lambda_{z}+\lambda_{x}\right) N_{z x}^{x},
$$


or, according to $(2.2 \mathrm{~b}),(1.1)$ and (1.3)

$$
\begin{aligned}
A(x, z) & =-\left(\lambda_{z}-\lambda_{x}\right)\left(\lambda_{x^{\prime}}-\lambda_{z}\right) \partial_{z} \lambda_{x^{\prime}}-\left(\lambda_{z}+\lambda_{x}\right)\left(\lambda_{x}-\lambda_{z}\right) \partial_{z} \lambda_{x} \\
& =-\left(\lambda_{z}-\lambda_{x}\right)\left(\lambda_{z}+\lambda_{x}\right) \partial_{z} \lambda_{x}+\left(\lambda_{z}+\lambda_{x}\right)\left(\lambda_{z}-\lambda_{x}\right) \partial_{z} \lambda_{x} \\
& \equiv 0
\end{aligned}
$$

Hence (4.5) is an identity regardless of whether $g \neq 0$ or $g=0$.

5. The vector $S_{\lambda}$. In this section as well as in the next one we shall deal only with the case of the first class. Moreover we shall assume also $g \neq 0$.

One of the field equations of the unified theory is

$$
S_{\lambda} \stackrel{\text { def }}{=} S_{\lambda \alpha}^{\alpha}=0
$$

Throughout this section as well as the next one we assume that (5.1) is satisfied.

Theorem (5.1). The condition (5.1) is equivalent to

$$
2 \lambda_{z} \lambda_{z z z^{\prime}}\left(S_{z x x^{\prime}}+S_{z x^{\prime} x}\right)=-N_{z z^{\prime} z}
$$

for all combinations of indices $z \neq x, x^{\prime}$.

Proof. Condition (5.1) is equivalent to

$$
S_{z x}^{x}+S_{z x^{\prime}}^{x^{\prime}}+S_{z z^{\prime}}^{z^{\prime}}=0 \quad\left(z \neq x, x^{\prime}\right),
$$

and this equation may be written by virtue of (1.5b)

$$
S_{z x x^{\prime}}+S_{z x^{\prime} x}+S_{z z^{\prime} z}=0 .
$$

On the other hand we have from (4.2)

$$
N_{z z^{\prime} z}=2 \lambda_{z} \lambda_{z z z^{\prime}} S_{z z^{\prime} z} \text {. }
$$

According to our assumptions we have $\lambda_{z} \lambda_{z z^{\prime} z} \neq 0$. Therefore we may substitute for $S_{z z^{\prime} z}$ from (5.4) into (5.3) and obtain (5.2) in this way.

Theorem (5.2). The equations (3.6a) and (5.1) admit a unique solution $S_{\lambda \mu}{ }^{\nu}$.

Proof. Equations (3.6) are equivalent to (4.1) and (4.2), and condition (5.1) is equivalent to (5.2). If we replace (4.1c) by the latter equation we obtain a system of three equations with the determinant

$$
\Delta=8\left(\lambda_{z} \lambda_{x x^{\prime} z}\right)^{2}\left(\lambda_{z}+\lambda_{x}\right) \lambda_{z z^{\prime} z} \neq 0^{*} \quad\left(z \neq x, x^{\prime}\right) .
$$

Hence this system yields $S_{i j k}(i j k \neq 0)$, while (4.2) gives the components $S_{x y x}$. This theorem will be needed in the next section.

\footnotetext{
* We have $\Delta \neq 0$ for all possible $x, z \neq x, x^{\prime}$ because $k_{\lambda \mu}$ is of the first class and $g \neq 0$.
} 


\section{Product space.}

Theorem (6.1). A necessary and sufficient condition that

(a) every pair of eigenvectors of $k_{\lambda}{ }^{\nu}$ be $X_{2}$-forming and

(b) every eigenvalue of $k_{\lambda}{ }^{\nu}$ be constant is

$$
S_{\lambda \mu}{ }^{\nu}=0 .
$$

Proof. Assume that (6.1) holds. Then (3.6a) yields

$$
N_{\lambda \mu}{ }^{\nu}=0 \text {. }
$$

Hence requirements (a) and (b) are satisfied by virtue of Theorems (2.2) and (2.3). Assume now that the requirements (a) and (b) are satisfied, so that (6.2) holds. Then the equations $(4.1 \mathrm{a}, \mathrm{b}),(5.2)$ and (4.2), together with condition (5.5), yield (6.1).

In the next theorem we denote by $V_{n}$ an $n$-dimensional Riemann space.

Theorem (6.2). Let the requirement (a) and (b) of the previous theorem be satisfied. Then our space-time is a $V_{4}$ which is a product space, $V_{4}=V_{2} \times V_{2}$. There is a coordinate system $e^{x}$ such that

$$
d s^{2}=h_{12}\left(e^{1}, e^{2}\right) d e^{1} d e^{2}+h_{34}\left(e^{3}, e^{4}\right) d e^{3} d e^{4}
$$

and the connection $\Gamma$ is given by

$$
\Gamma_{x x}^{x}=\left\{\begin{array}{c}
x \\
x x
\end{array}\right\}=\partial_{x} \ln h_{x x^{\prime}}
$$

$$
\text { the remaining } \Gamma_{x y}^{z}=0 \text {. }
$$

Proof. Assume that requirement (a) of the previous theorem is satisfied. Then there is a coordinate system $e^{x}$ in which the eigenvectors satisfy the conditions

$$
{ }_{j} e^{\nu}=\frac{\partial x^{\nu}}{\partial e^{j}}, \quad{ }^{i} e_{\lambda}=\frac{\partial e^{i}}{\partial x^{\lambda}} .
$$

These eigenvectors satisfy (1.5a), where

$$
h_{i j}=h_{j i}=\left(\begin{array}{cccc}
0 & h_{12} & 0 & 0 \\
h_{21} & 0 & 0 & 0 \\
0 & 0 & 0 & h_{34} \\
0 & 0 & h_{43} & 0
\end{array}\right), \quad h_{12} h_{34} \neq 0 .
$$

Assume now that both requirements (a) and (b) of the previous theorem hold. Then the eigenvalues involved in

$$
k_{x y}=\lambda_{x} h_{x y}{ }^{*}
$$

\footnotetext{
* This equation follows at once from (1.7).
} 
are constant. Moreover from (6.1) and (1.19) we have

$$
\Gamma_{\lambda \mu}^{\nu}=\left\{\begin{array}{c}
\nu \\
\lambda \mu
\end{array}\right\}
$$

and, according to Theorem I, (8.1) in [3]

$$
K_{\omega \mu \nu}=0 \text {. }
$$

Therefore by virtue of (1.18)

$$
K_{\mu[\nu \omega]}=\nabla_{\mu} k_{\omega \nu}=0 .
$$

Consequently by virtue of (6.7)

$$
\begin{aligned}
0= & \nabla_{x} k_{y z}+\lambda_{z} \nabla_{x} h_{y z} \\
= & \lambda_{y} \partial_{x} h_{y z}-\left\{\begin{array}{c}
z^{\prime} \\
y x
\end{array}\right\} \lambda_{z^{\prime}} h_{z^{\prime} z}-\left\{\begin{array}{l}
y^{\prime} \\
z x
\end{array}\right\} \lambda_{y} h_{y y^{\prime}} \\
& +\lambda_{z}\left(\partial_{x} h_{y z}-\left\{\begin{array}{c}
z^{\prime} \\
y x
\end{array}\right\} h_{z^{\prime} z}-\left\{\begin{array}{l}
y^{\prime} \\
z x
\end{array}\right\} h_{y y^{\prime}}\right) \\
= & \left(\lambda_{y}+\lambda_{z}\right)\left[\partial_{x} h_{y z}-\left\{\begin{array}{l}
y^{\prime} \\
z x
\end{array}\right\} h_{y y^{\prime}}\right] .
\end{aligned}
$$

On the other hand we have

$$
\lambda_{y}+\lambda_{z} \neq 0
$$

for $z \neq y^{\prime}$,

so that according to $(6.10)$

$$
\left\{\begin{array}{l}
y^{\prime} \\
x z
\end{array}\right\}=\left\{\begin{array}{l}
y^{\prime} \\
z x
\end{array}\right\}=0 \quad \text { for } z \neq y^{\prime *} .
$$

Equation (6.11) yields for $y=x^{\prime}, z \neq x$

$$
\left\{\begin{array}{c}
x \\
z x
\end{array}\right\}=\left\{\begin{array}{c}
x \\
x z
\end{array}\right\}=0, \quad z \neq x .
$$

On the other hand according to (6.12)

$$
\left\{\begin{array}{c}
y^{\prime} \\
z x
\end{array}\right\}=\left\{\begin{array}{c}
z \\
z x
\end{array}\right\}=0 \quad \text { for } z=y^{\prime}, z \neq x .
$$

* One must bear in mind that in our case

$$
\left\{\begin{array}{c}
y^{\prime} \\
{[x y]}
\end{array}\right\}=0
$$





\section{Degenerate Cases}

7. First class. In this second part we shall deal with solutions of (1.20) in exceptional cases, characterized by (0.2). In this section we consider the exceptional case of the first class, characterized by

$$
\begin{gathered}
k D \neq 0, \\
g(1)=1+2 K+k=0 .
\end{gathered}
$$

Lemma (7.1). We have*

$$
\begin{gathered}
\alpha=\lambda_{1}=-\lambda_{2}=-\sqrt{k} \\
\beta=\lambda_{3}=-\lambda_{4}=1
\end{gathered}
$$

and

$$
\begin{aligned}
& \text { a) } \quad \lambda_{84 i}=0 \\
& \text { b) } \quad \lambda_{12 i}=1-k \\
& \text { c) } \quad \lambda_{\text {aff }}=2(1-\epsilon \sqrt{k}) \\
& \text { d) } \quad \lambda_{a a f}=(1-\epsilon \sqrt{k})^{2} \\
& \left(\epsilon=(-1)^{a+f}\right) .
\end{aligned}
$$

Proof. The equations (7.2) follow at once from (1.2) (1.3) and (7.1). The equations (7.3) follow by simple inspection from (1.17) and (7.2).

In order to prove the next theorems we need the following two lemmas, which will be proved simultaneously.

Lemma (7.2). We have

$$
\nabla_{i} k_{f e}=0
$$

and

$$
\begin{gathered}
\left\{\begin{array}{c}
a^{\prime} \\
{[e f]}
\end{array}\right\}+\frac{1}{2}\left\{\begin{array}{c}
e^{\prime} \\
a f
\end{array}\right\}-\frac{1}{2}\left\{\begin{array}{c}
f^{\prime} \\
a e
\end{array}\right\}=0 \\
\left\{\begin{array}{c}
a^{\prime} \\
(e f)
\end{array}\right\}+\frac{1}{2}\left\{\begin{array}{c}
e^{\prime} \\
a f
\end{array}\right\}+\frac{1}{2}\left\{\begin{array}{c}
f^{\prime} \\
a e
\end{array}\right\}=0
\end{gathered}
$$

Lemma (7.3). The following equation holds:

$$
K_{i e f}=K_{i(e f)} .
$$

\footnotetext{
* For the first class we have $k<0$.
} 
Proof: First of all, according to (1.7) we have

$$
k_{e f}=\lambda_{e} h_{e f}\left(=-\lambda_{f} h_{e f}\right),
$$

so that

$$
\begin{aligned}
\nabla_{i} k_{e f} & =\partial_{i} k_{e f}-\left\{\begin{array}{l}
f^{\prime} \\
e i
\end{array}\right\} k_{f^{\prime} f}-\left\{\begin{array}{l}
e^{\prime} \\
f i
\end{array}\right\} k_{e e^{\prime}} \\
& =\lambda_{e} \partial_{i} h_{e f}-\left\{\begin{array}{l}
f^{\prime} \\
e i
\end{array}\right\} \lambda_{f^{\prime}} h_{f^{\prime} f}-\left\{\begin{array}{l}
e^{\prime} \\
f i
\end{array}\right\} \lambda_{e} h_{e e^{\prime}} \\
& =\lambda_{e}\left[\partial_{i} h_{e f}-\left\{\begin{array}{l}
f^{\prime} \\
e i
\end{array}\right\} h_{f^{\prime} f}-\left\{\begin{array}{l}
e^{\prime} \\
f i
\end{array}\right\} h_{e e^{\prime}}\right]=\lambda_{e} \nabla_{i} h_{e f}=0
\end{aligned}
$$

Moreover

$$
\begin{aligned}
0=2 \nabla_{[f} h_{e] a} & =-2\left[\left\{\begin{array}{c}
a^{\prime} \\
{[e f]}
\end{array}\right\} h_{a a^{\prime}}+\left\{\begin{array}{c}
j \\
a[f
\end{array}\right\} h_{e] j}\right] \\
& =-2\left[\left\{\begin{array}{c}
a^{\prime} \\
{[f e]}
\end{array}\right\}+\frac{1}{2}\left\{\begin{array}{c}
e^{\prime} \\
a f
\end{array}\right\}-\frac{1}{2}\left\{\begin{array}{c}
f^{\prime} \\
a e
\end{array}\right\}\right]
\end{aligned}
$$

and

$$
\begin{aligned}
0=2 \nabla\left(f h_{e) a}\right. & =-2\left[\left\{\begin{array}{c}
a^{\prime} \\
(f e)
\end{array}\right\} h_{a^{\prime} a}+\left\{\begin{array}{c}
j \\
a(f)
\end{array}\right\} h_{e) j}\right] \\
& =-2\left[-\left\{\begin{array}{c}
a^{\prime} \\
(f e)
\end{array}\right\}+\frac{1}{2}\left\{\begin{array}{c}
e^{\prime} \\
a f
\end{array}\right\}+\frac{1}{2}\left\{\begin{array}{c}
f^{\prime} \\
a e
\end{array}\right\}\right] .
\end{aligned}
$$

The equation (7.5c) follows at once from (7.4) and (1.18).

The following two theorems will be proved simultaneously.

Theorem (7.1a). The following equation holds:

$$
k_{(\omega}{ }^{\nu} K_{\mu) \lambda \nu} P^{\omega \mu}(\alpha)=0 ; *
$$

it is equivalent to

$$
K_{\lambda \omega \mu} Q^{\omega \mu}(\alpha)=0 .
$$

Theorem (7.1b). A necessary and sufficient condition for (1.20) to admit at least one solution $S_{\lambda \mu}{ }^{\nu}$ is

$$
k_{(\lambda}{ }^{\nu} K_{\omega) \mu \nu} Q^{\omega \mu}(\alpha)=0 .
$$

Proof. The scalar $k$ being negative, it follows from (7.3) that the only scalars $\lambda_{i j k}$ which are equal to zero are $\lambda_{34 i}$. Hence the system $(1.21 \mathrm{a}, \mathrm{b}, \mathrm{c})$ for $x=e$,

* $C f .(1.8)$ and (1.13). 
$y=f, z=a$ cannot be solved uniquely for the $S$ 's, its determinant being equal to zero. This system reduces by virtue of $(7.3 \mathrm{a})$ to

$$
\begin{aligned}
& \frac{1}{2} K_{e f a}=-S_{e f a} \lambda_{e} \lambda_{f}-S_{f a e} \lambda_{e} \lambda_{f}-S_{e a f} \lambda_{e} \lambda_{f} \\
& \frac{1}{2} K_{f a e}=-S_{e f a} \lambda_{f} \lambda_{a}-S_{f a e} \lambda_{f} \lambda_{a}-S_{e a f} \lambda_{f} \lambda_{a} \\
& \frac{1}{2} K_{a e f}=-S_{e f a} \lambda_{a} \lambda_{e}-S_{f a e} \lambda_{a} \lambda_{e}-S_{e a f} \lambda_{a} \lambda_{e},
\end{aligned}
$$

so that a necessary and sufficient condition that (7.9) admit at least one solution is obviously

$$
K_{e f a} \lambda_{a}=K_{f a e} \lambda_{e}=K_{a e f} \lambda_{f}
$$

On the other hand the right hand side of (1.21a) vanishes for $x=e, y=f, z=f$. Hence we must also have

$$
K_{\text {eff }}=0 .
$$

Equation $(7.10 \mathrm{a}, \mathrm{b})$ can be condensed into

$$
K_{e f i} \lambda_{i}=K_{f i e} \lambda_{e}=K_{i e f} \lambda_{f}
$$

Some of these conditions are satisfied as we shall see later.

First let us consider the last of equations (7.10c) which, by virtue of (1.7), may be written as follows:

$$
\begin{aligned}
0 & =K_{f i j} k_{e}^{j}-K_{i e j} k_{f}^{j} \\
& =2 k_{(e}^{j} K_{f) i j .}
\end{aligned}
$$

On the other hand we may rewrite the same equation in another form resulting from $(7.2 \mathrm{~b})$ :

$$
0=K_{f i e}+K_{i e f}=2 K_{i[e f]} .
$$

Remembering (1.9) and (1.14), we see that (7.11a, b) are equivalent to (7.6) and (7.7). On the other hand (7.11b) is satisfied by virtue of (7.5c). The first equation $(7.10 \mathrm{c})$ may be rewritten as

$$
\begin{aligned}
0 & =K_{e f i} \lambda_{i}-\frac{1}{2}\left(K_{f i e} \lambda_{e}+K_{i e f} \lambda_{f}\right) \\
& =K_{e f j} k_{i}^{j}-k_{[e}^{j} K_{f] i j},
\end{aligned}
$$

or, by virtue of (1.14),

$$
\begin{aligned}
0 & =Q^{\omega \mu}(\alpha)\left(K_{\omega \mu \nu} k_{\lambda}{ }^{\nu}-k_{\omega}{ }^{\nu} K_{\mu \lambda \nu}\right) \\
& =2 Q^{\omega \mu}(\alpha) k_{(\lambda}{ }^{\nu} K_{\omega) \mu \nu} .
\end{aligned}
$$


Remark. The last term in (1.19a) is

$$
U_{\lambda \mu}^{\nu} \stackrel{\text { def }}{=} 2 h^{\alpha \nu} S_{\alpha(\lambda}^{\beta} k_{\mu) \beta} .
$$

A necessary and sufficient condition for $U_{\lambda \mu}^{\nu}=0$ is

$$
k_{(\lambda}{ }^{\nu} K_{\omega) \mu \nu}=0 \quad \text { (cf. Theorem (9.2a) in [1]). }
$$

If this condition is satisfied, then the conditions (7.6) and (7.8) are identically satisfied. However we cannot use this result, for the necessary and sufficient condition mentioned above has been derived under assumptions (1.23), the first of which contradicts (7.1b).

Theorem (7.2). Whenever (7.8) holds, the eigenvectors ${ }_{3} e^{\lambda},{ }_{4} e^{\nu}$ are $X_{2}$-forming.

Proof. Whenever (7.8) holds, the system (1.21) admits at least one solution. Therefore, by virtue of $(1.19 \mathrm{~b})$, the system (0.1) admits at least one solution and consequently, according to Theorem (3.4), the relationship (3.6) exists. Therefore the system (4.1) exists. For $x=e, x^{\prime}=f, z=a$ this system reduces, according to (7.3), to
a) $N_{e f}^{a^{\prime}}=0$
b) $N_{a f}^{f}=0$
c) $N_{a e}^{e}=0$.

The equations $(7.12 \mathrm{~b}, \mathrm{c})$ confirm the fact that $\lambda_{3}, \lambda_{4}$ are constant ( $c f$. Theorem (2.3)). Equation (7.12a) asserts, by virtue of Theorem (2.2), that the eigenvectors ${ }_{3} e^{\lambda},{ }_{4} e^{\nu}$ are $X_{2}$-forming, i.e.

$$
\left\{\begin{array}{c}
a \\
{[e f]}
\end{array}\right\}=0^{*} \quad e \neq f .
$$

Theorem (7.3). A necessary and sufficient condition for the system (1.20) to admit at least one solution is that the eigenvectors ${ }_{3} e^{\lambda},{ }_{4} e^{\nu}$ are $X_{2}$-forming.

Proof. The necessity follows at once from Theorems (7.1b) and (7.2). Assume now that ${ }_{3} e^{\lambda},{ }_{4} e^{\nu}$ are $X_{2}$-forming, so that (7.13) holds. Therefore the equations (7.5) reduce to

$$
\begin{gathered}
\left\{\begin{array}{c}
f \\
a f
\end{array}\right\}=\left\{\begin{array}{c}
e \\
a e
\end{array}\right\} \\
2\left\{\begin{array}{c}
a^{\prime} \\
e f
\end{array}\right\}+\left\{\begin{array}{c}
f \\
a f
\end{array}\right\}+\left\{\begin{array}{c}
e \\
a e
\end{array}\right\}=0,
\end{gathered}
$$

* As a matter of fact we have $k \neq \pm 1$, and therefore (7.12a) is equivalent to

$$
\left\{\begin{array}{c}
a^{\prime} \\
{[e f]}
\end{array}\right\}=0,
$$

and this equation is equivalent to (7.13), since the range of $a$ and $a^{\prime}$ is the same, namely $1,2$. 
so that according to (7.4)

$$
\begin{aligned}
\lambda_{a} K_{e f a}-\lambda_{e} K_{f a e} & =\left(\lambda_{a}+\lambda_{e}\right) \nabla_{e} k_{a f}+\left(\lambda_{a}-\lambda_{e}\right) \nabla_{f} k_{e a} \\
& =-\lambda_{e}\left\{\lambda_{a}\left[2\left\{\begin{array}{c}
a^{\prime} \\
e f
\end{array}\right\}+\left\{\begin{array}{c}
e \\
a e
\end{array}\right\}+\left\{\begin{array}{c}
f \\
a f
\end{array}\right\}\right]+\lambda_{e}\left[\left\{\begin{array}{c}
e \\
a e
\end{array}\right\}-\left\{\begin{array}{c}
f \\
a f
\end{array}\right\}\right]\right\} \\
& =0 .
\end{aligned}
$$

Hence the first equation (7.10c) for $i=a$ is satisfied. The remaining equations (7.10c) are satisfied by virtue of Theorem (7.1a).

\section{First class. Continuation.}

Theorem (8.1). Let $k_{\mu \lambda}$ be of the first class and assume that (7.1) holds and that the eigenvectors ${ }_{3} e^{\lambda},{ }_{4} e^{\nu}$ are $X_{2}$-forming. Then the system (0.1) admits at least one solution (1.19a). Its skew symmetric part $S_{\lambda \mu}{ }^{\nu}$ has the following non-holonomic components:

(a) The components $S_{\text {eff }}$ are arbitrary;

(b) The components $S_{e f a}, S_{f a e}, S_{a e f}$ are arbitrary but for the single condition

$$
\frac{1}{2} K_{e f a}=3 S_{[e f a]}^{*}
$$

(c) The remaining components are

$$
\begin{aligned}
& S_{f a b}=\frac{1}{2 \lambda_{f a b}}\left[K_{f a b}+3 \lambda_{f} \lambda_{a} K_{[f a b]}\right] \\
& S_{a b f}=\frac{1}{2 \lambda_{f a b}}\left[K_{a b f}+3 \lambda_{a} \lambda_{b} K_{[a b f]}\right] \\
& S_{a b c}=\frac{1}{2 \lambda_{a b c}} K_{a b c} \\
& S_{e a e}=\frac{1}{2 \lambda_{e e a}} K_{e a e},
\end{aligned}
$$

where the $\lambda$ 's are to be taken from (7.2) and (7.3).

Proof. According to previous theorems, the system (1.20) admits at least one solution. The right hand side of (1.21a) vanishes for $x=e, y=f=z$ so that this equation does not yield $S_{\text {eff. }}$. There is no other equation among (1.21) yielding $S_{\text {eff }}$. Hence $S_{\text {eff }}$ are not defined by (1.21). Equation (8.1) follows at once from the first equation of (7.9). The remaining components given by (8.2) result from (1.21d).

* Using (7.2b), (7.3a) and (7.10c) we easily obtain for $e \neq f$

$$
3 K_{[e f i]}=K_{e f i}\left(1+\frac{\lambda_{i}}{\lambda_{e}}+\frac{\lambda_{i}}{\lambda_{f}}\right)=\frac{\lambda_{e f i}-1}{\lambda_{e} \lambda_{f}} K_{e f i}=K_{e f i} .
$$


Remark. Put $x=f, y=e$ in (4.2):

$$
N_{f e f}=N_{f e}^{\bullet}=-2 \lambda_{f} \lambda_{f f e} S_{e f f}
$$

The left term vanishes for $\lambda_{3}=-\lambda_{4}=$ const. (cf. Theorem (2.3)). The right term vanishes for $\lambda_{f f e}=0$. Hence not even (3.6) can be used for finding $S_{\text {eff }}$.

In the non-degenerate case equation (5.1) imposes some conditions on $g_{\lambda \mu}$. In our degenerate case the requirement (5.1) does not impose any condition on $g_{\lambda \mu}$ :

Theorem (8.2). There is a two-parametric set of solutions $\Gamma_{\lambda \mu}^{\nu}$, mentioned in the previous theorem, which satisfy (5.1). Its skew symmetric part $S_{\lambda \mu}{ }^{\nu}$ has the following components:

(a) The components $S_{\text {eff }}$ are given by

$$
S_{e f f}=\frac{1}{1-k}\left[K_{f(a b)}+3 \lambda_{a} \lambda_{f} K_{[f a b]}\right], \quad a \neq b, \quad e \neq f
$$

(b) Two components, namely $S_{\text {efa }}$, are arbitrary while

$$
2 S_{a e f}=\frac{1}{2} K_{e f a}-\frac{1}{2(1-k)} K_{a b a}-S_{e f a} ;
$$

(c) The remaining components are given by (8.2).

Proof. Equation (5.1) is equivalent to

$$
\begin{aligned}
& S_{f e f}+S_{f a b}+S_{f b a}=0 \\
& S_{a b a}+S_{a f e}+S_{a e f}=0
\end{aligned}
$$$$
a \neq b, e \neq f
$$

From (8.4a) and (8.2a) we obtain for $a \neq b, e \neq f$

$$
\begin{aligned}
S_{\text {eff }} & =\frac{1}{2 \lambda_{f a b}}\left[2 K_{f(a b)}+3 \lambda_{a} \lambda_{f} K_{[f a b]}+3 \lambda_{b} \lambda_{f} K_{[f b a]}\right] \\
& =\frac{1}{2(1-k)}\left[2 K_{f(a b)}+6 \lambda_{a} \lambda_{f} K_{[f a b]}\right] .
\end{aligned}
$$

On the other hand (8.4b) and (8.1) yield

$$
2 S_{a e f}=\frac{1}{2} K_{e f a}-S_{a b a}-S_{e f a}
$$$$
a \neq b, \quad e \neq f
$$

Substituting from (8.2c) into this equation one obtains (8.3b).

9. Second class: The first case. The second class is characterized by the condition

$$
D=K^{2} \neq 0, \quad k=0 .
$$

There are two degenerate cases, namely

$$
g(1)=g=0
$$




$$
g(1)=g=2 \text {. }
$$

In this section we consider the case (9.1a) and prove first the following

Lemma (9.1). We have

$$
\begin{aligned}
& \alpha=\lambda_{1}=-\lambda_{2}=0 \\
& \beta=\lambda_{3}=-\lambda_{4}=1
\end{aligned}
$$

and
a) $\lambda_{34 i}=0$
b) $\lambda_{12 i}=\lambda_{\text {aaf }}=1$,
c) $\lambda_{\text {aff }}=2$.

Proof. Equations (7.2), (7.3) hold for any value of $k$ which satisfies (7.1b) (i.e., (9.1a)). Hence they hold also for $k=0$ and reduce in this case to (9.3).

The following two theorems will be proved simultaneously.

Theorem (9.1a). We have

$$
K_{\omega \mu \lambda} Q^{\omega \mu}(\alpha) P^{\lambda \nu}(\alpha)=0 .
$$

Theorem (9.1b). A necessary and sufficient condition for (1.20) to admit at least one solution is

$$
K_{\omega \mu \lambda} P^{\mu \lambda}(\alpha) P^{\omega \nu}(\beta)=0 .
$$

Proof. A simple inspection shows that the required necessary and sufficient conditions may be written in the form $(7.10 \mathrm{c})$ for $\lambda_{a}=0$ so that by virtue of (7.4) they are equivalent to

$$
0=K_{i e f}=-2 \nabla_{(e} k_{f) i}=K_{i(e f)} \quad(e \neq f) .
$$

For $i=f$ we obtain from (9.5) equation (7.10b) which is satisfied because of (7.4). On the other hand (7.10b) may be written in the form (9.4a). Hence the remaining necessary and sufficient conditions reduce to (9.5) for $i=a$, and this system is obviously equivalent to $(9.4 \mathrm{~b})$.

Theorem (9.2). A necessary and sufficient condition that (1.20) admit at least one solution $S_{\lambda \mu}{ }^{\nu}$ is that the eigenvectors ${ }_{3} e^{\lambda},{ }_{4} e^{\nu}$ are $X_{2}$-forming.

The proof of necessity follows the same pattern as the proof of Theorem (7.2). Assume now that ${ }_{3} e^{\nu},{ }_{4} e^{\lambda}$ are $X_{2}$-forming so that (7.13) holds. Therefore from (7.4), (7.5) and (9.2a) we derive for $e \neq f$

$$
\begin{aligned}
K_{f a e}=2 \nabla_{(f} k_{e) a} & =-\left[\left\{\begin{array}{c}
f \\
a f
\end{array}\right\} k_{e f}+\left\{\begin{array}{c}
e \\
a e
\end{array}\right\} k_{f e}\right] \\
& \left.=-k_{e f}\left[\begin{array}{c}
f \\
a f
\end{array}\right\}-\left\{\begin{array}{c}
e \\
a e
\end{array}\right\}\right]=0 .
\end{aligned}
$$

These equations together with (7.10b) are condensed in (9.5).

Theorem (9.3). Let $k_{\mu \lambda}$ be of the second class and assume that (9.1a) holds and 
that the eigenvectors ${ }_{3} e^{\lambda},{ }_{4} e^{\nu}$ are $X_{2}$-forming. Then the system (0.1) admits at least one solution (1.19a). Its skew symmetric part $S_{\lambda \mu}{ }^{\nu}$ has the following non-holonomic components:

(a) the components $S_{\text {eff }}$ are arbitrary;

(b) the components $S_{\text {efa }}, S_{\text {fae }}$, $S_{a e f}$ are arbitrary but for the single condition

$$
\frac{1}{2} K_{e f a}=3 S_{[e f a]} *
$$$$
(e \neq f) \text {; }
$$

(c) the remaining components are

$$
\begin{aligned}
& S_{f a b}=\frac{1}{2} K_{f a b}, \\
& S_{a b f}=\frac{1}{2} K_{a b f}, \\
& S_{a b c}=0, \\
& S_{e a e}=\frac{1}{4} K_{e a e} .
\end{aligned}
$$

The proof follows the pattern of the proof of Theorem (8.1). As far as (9.7c) is concerned one must keep in mind that, according to (1.7), (1.5b) and (9.2), the only component $k_{i j}$ not equal to zero is $k_{34}=-k_{43}$. Hence we have

$$
\nabla_{a} k_{b c}=0
$$

and therefore also $K_{a b c}=0$. This equation together with (8.2c) and (9.3b) leads to $(9.7 \mathrm{c})$.

Theorem (9.4). There is a two-parametric set of solutions $\Gamma_{\lambda_{\mu}}^{\nu}$, mentioned in the previous theorem, which satisfy (5.1). Its skew symmetric part $S_{\lambda \mu}{ }^{\nu}$ has the following components:

(a) the components $S_{\text {eff }}$ are given by

$$
S_{e f f}=K_{f(a b)}
$$$$
e \neq f, \quad a \neq b
$$

(b) the two components $S_{\text {efa }}$ are arbitrary while

$$
2 S_{a e f}=\frac{1}{2} K_{e f a}-S_{e f a}
$$

(c) the remaining components are given by (9.7).

The proof follows the pattern of the proof of Theorem (8.2).

10. Second class: The second case. The second degenerate case of the second class is characterized by the conditions

$$
D=K^{2} \neq 0, \quad k=0
$$

* From (9.5) we have

$$
K_{e f a}=3 K_{[e f a]} .
$$


and $(9.1 \mathrm{~b})$, i.e.

$$
g(1)=g=2 .
$$

In this section we shall consider this case.

Lemma (10.1). We have

$$
\begin{aligned}
& \alpha=\lambda_{1}=-\lambda_{2}=i \\
& \beta=\lambda_{3}=-\lambda_{4}=0 .
\end{aligned}
$$

and

$$
\begin{aligned}
& \lambda_{12 i}=2 \\
& \lambda_{34 i}=\lambda_{a f f}=1 \\
& \lambda_{a a f}=0 .
\end{aligned}
$$

Proof. Equations (10.2) follow at once from (1.2) (1.3) and (10.1) because the last of these equations is equivalent to $2 K=1$. The remaining equations follow from (1.17) and (10.2).

Theorem (10.1). A necessary and sufficient condition for the system (1.20) to admit at least one solution $S_{\lambda \mu \nu}$ is

$$
K_{\text {faa }}=0 .
$$

Proof. According to (10.3) the determinant $\lambda^{2}{ }_{x y z}$ of (1.21) (for $x, y, z \neq$ ) is different from zero. Hence this system does not impose any condition on $k_{\lambda \mu}$. Moreover according to (10.2) and (10.3) it is obvious that the only possible restriction imposed upon $k_{\lambda \mu}$ might result from (1.21a) for $x=f, y=z=a$. This restriction is (10.4), which obviously is not only necessary but also sufficient for the system (1.21) (equivalent to (1.20)) to admit at least one solution.

In the next theorem we denote by the name "the trajectories of $e_{a} e^{\nu "}$ the curves with the tangential vector ${ }_{a} e^{\nu}$.

Theorem (10.2). A necessary and sufficient condition for the system (1.20) to admit at least one solution is that the trajectories of ${ }_{a} e^{\nu}$ are geodesic lines.

Proof. First of all we have

$$
\begin{aligned}
K_{f a a}=K_{f a a}+2 \lambda_{a} \nabla_{a} h_{f a} & =2\left[\nabla_{a} k_{f a}+\lambda_{a} \nabla_{a} h_{f a}\right] & \\
& =-2 \lambda_{a}\left\{\begin{array}{c}
e \\
a a
\end{array}\right\} & (e \neq f) .
\end{aligned}
$$

Hence we see from (1.24) that (10.4) is equivalent to

$$
{ }_{a} e^{\lambda}\left(\nabla_{\lambda} e^{\mu}\right)^{e} e_{\mu}=0 \text {. }
$$

\footnotetext{
* Equation (1.21b) reduces to the identity $0=0$, while $(1.21 \mathrm{c})$ reduces to (1.21a).
} 
Assume now that the trajectories of ${ }_{a} e^{\nu}$ are geodesic lines

$$
{ }_{a} e^{\lambda} \nabla_{\lambda a} e^{\nu}=\rho_{a} e^{\nu} .
$$

Then (10.6) is satisfied, and therefore, by virtue of (10.5), (10.4) is also satisfied. Assume now that (10.4) is satisfied. Then we see from (10.5) that (10.6) holds, and this condition is equivalent to

$$
{ }_{a} e^{\lambda} \nabla_{\lambda a} e^{\nu}=\rho_{a a} a e^{\nu}+\rho_{a b} b e^{\nu} \quad(a \neq b),
$$

so that

$$
\begin{aligned}
\rho_{a b b} e^{\nu b} e_{\nu}=\rho_{a b} & ={ }_{a} e^{\lambda}\left(\nabla_{\lambda_{a}} e^{\nu}\right)^{b} e_{\nu} \\
& ={ }_{a} e^{\lambda}\left(\nabla_{\lambda_{a}} e^{\nu}\right)_{a} e_{\nu} \\
& ={ }_{a} e^{\lambda}\left(\nabla_{\lambda_{a}} e^{\mu}\right)_{a} e^{\nu} h_{\mu \nu} .
\end{aligned}
$$

The last term is obviously equal to zero, for ${ }_{a} e^{\lambda}$ is a null vector. Hence we have $\rho_{a b}=0$, and (10.8) reduces to (10.7) (for $\rho_{a}=\rho_{a a}$ ).

Theorem (10.3). A sufficient condition for the system (1.20) to admit at least one solution is that the eigenvectors ${ }_{a} e^{\nu}$ and $e_{f}^{\nu}$ are $X_{2}$-forming.

Proof. Assume that ${ }_{a} e^{\nu}$ and ${ }_{f} e^{\nu}$ are $X_{2}$-forming:

$$
\left\{\begin{array}{c}
j \\
{[a f]}
\end{array}\right\}=0 \quad(j \neq a, f) .
$$

A simple inspection shows that for $b \neq a$

$$
K_{f a a}=K_{f a a}+\lambda_{a} \nabla_{f} h_{a a}=4 \lambda_{a}\left\{\begin{array}{c}
b \\
{[f a]}
\end{array}\right\} \text {. }
$$

Hence if (10.9a) is satisfied, so is (10.4).

Remark. If we substitute into (4.2) $x=a, y=f$, and $x=f, y=a$ respectively, we obtain, according to (10.3c) and (10.2b),

$$
\begin{aligned}
& N_{a f}^{b}=0 \\
& N_{f a}{ }^{e}=0,
\end{aligned}
$$$$
a \neq b, \quad e \neq f .
$$

However these equations are not sufficient for ${ }_{f} e^{\nu}$ and ${ }_{a} e^{\nu}$ to be $X_{2}$-forming, since condition (2.3a) is not satisfied for $z=e, x=f, y=a$.

Theorem (10.4). Let $k_{\lambda_{\mu}}$ be of the second class and assume that (10.1) holds and that the trajectories of ${ }_{a} e^{v}$ are geodesic lines. Then the system (0.1) admits at least one solution (1.19a). Its skew symmetric part $S_{\lambda \mu}{ }^{\nu}$ has the following non-holonomic components:

(a) the four components $S_{f a a}$ are arbitrary;

(b) the remaining components are

$$
S_{e f o}=S_{a b c}=0
$$


$(10.10 \mathrm{~b})$

$$
\begin{aligned}
& S_{e f a}=\frac{1}{2} K_{e f a} \\
& S_{a b f}=\frac{1}{4}\left(K_{a b j}+3 K_{[a b j]}\right), \\
& S_{a f b}=\frac{1}{4} K_{a f b} \\
& S_{a f g}=\frac{1}{2} K_{a f g} .
\end{aligned}
$$

Proof. Here we have

$$
\nabla_{i} k_{e f}=0, \quad \nabla_{j} k_{a b}=\lambda_{a} \nabla_{j} h_{a b}=0,
$$

and therefore

$$
K_{\text {ef } \theta}=0, \quad K_{a b c}=0 .
$$

The remaining steps of the proof follow a pattern similar to the one used in the proof of Theorem (8.1).

Remark. The equations (10.10a) may be condensed into one set:

$$
Q^{\omega \mu}(\gamma) S_{\omega \mu}{ }^{\lambda} P_{\lambda}{ }^{\nu}(\gamma)=0 .
$$

Contrary to the situation in the previous degenerate cases, condition (5.1) now imposes a restriction on $g_{\lambda \mu}$. The next two theorems, which describe this situation, will be proved simultaneously.

Theorem (10.5). A necessary and sufficient condition that the solution described in Theorem (10.4) satisfies (5.1) is

$$
P_{\omega}{ }^{\nu}(\gamma) K_{\nu \lambda \mu} P^{\lambda \mu}(\alpha+\beta-\gamma)=0 .
$$

If this condition is satisfied then

$$
S_{a f b}=0, \quad S_{a f e}=0, \quad S_{a b f}=\frac{1}{2} K_{a b f} \quad(a \neq b, \quad e \neq f),
$$

while the remaining components of $S$ remain the same as in Theorem (10.4).

Theorem (10.6). Condition (10.11) for $\gamma=\alpha$ is equivalent to the requirement that the eigenvectors ${ }_{1} e^{v}$ and ${ }_{2} e^{\nu}$ are $X_{2}$-forming.

Proof. Here we have for $a \neq b$

$$
K_{f a b}=\nabla_{f} k_{b a}+\nabla_{a} k_{f b}+\nabla_{b} k_{f a}=-2 \nabla_{(a} k_{b) f}=K_{f(a b),}
$$

and moreover

$$
K_{f a b}=K_{f a b}-\left(\lambda_{a} \nabla_{a} h_{f b}+\lambda_{b} \nabla_{b} h_{f a}\right)=2 \lambda_{a}\left\{\begin{array}{c}
e \\
{[b a]}
\end{array}\right\} \quad(e \neq f) .
$$

Hence condition (10.12a) for $\gamma=\alpha$ is equivalent to

$$
\left\{\begin{array}{c}
e \\
{[b a]}
\end{array}\right\}=0
$$


so that ${ }_{1} e^{\nu}$ and ${ }_{2} e^{\nu}$ are $X_{2}$-forming. The condition (5.1) is equivalent to

$$
\begin{aligned}
& S_{f a b}+S_{f b a}+S_{f e f}=0 \\
& S_{a f e}+S_{a e f}+S_{a b a}=0, \quad a \neq b, \quad e \neq f,
\end{aligned}
$$

or according to $(10.10)$ and $(10.13 \mathrm{~b})$

$$
\begin{aligned}
& 0=-S_{f e f}=2 S_{f(a b)}=\frac{1}{2} K_{f(a b)}=\frac{1}{2} K_{f a b} \\
& 0=-S_{a b a}=2 S_{a(f e)}=K_{a(f e)}=K_{a f e}, \quad a \neq b, \quad e \neq f .
\end{aligned}
$$

The conditions (10.14) are equivalent to (10.12a). Substituting from (10.14) into $(10.10 \mathrm{c}, \mathrm{d}, \mathrm{e})$ we obtain $(10.12 \mathrm{~b})$.

\section{BIBLIOGRAPHY}

[1] Huavatý, VÁclav, "The Elementary Basic Principles of the Unified Theory of Relativity. B," Journal of Rational Mechanics and Analysis, Vol. 2, No. 1, January, 1953, pp. 1-52.

[2] Hlavaț́, VÁclav, \& Sáenz, A. W., "Uniqueness Theorems in the Unified Theory of Relativity," Journal of Rational Mechanics and Analysis, Vol. 2, No. 3, July, 1953, pp. 523-536.

[3] Hlavatý, Víclav, "The Elementary Basic Principles of the Unified Theory of Relativity. $\mathrm{C}_{1}$ : Introduction," Journal of Rational Mechanics and Analysis, Vol. 3, No. 1, January, 1954, pp. 103-146.

[4] HlavatÝ, VÁclav, "The Elementary Basic Principles of the Unified Theory of Relativity. $\mathrm{B}_{2}$ " Journal of Rational Mechanics and Analysis, Vol. 4, No. 2, March, 1954, pp. 247-277.

[5] NiJenhuis, Albert, " $X_{n-1}$-forming sets of eigenvectors," Koninklijke Nederlandse Akademie van Wetenschappen, Proceedings, Series A, Vol. LIV, No. 2, 1951, pp. 200-212.

* We have

$$
K_{a f e}=\nabla_{a} k_{e f}+\nabla_{f} k_{a e}+\nabla_{e} k_{a f}=-2 \nabla_{(f} k_{e) a}=K_{a(f e)} .
$$

